\title{
Performance Evaluation of Wireless Sensor Networks Based on Entropy Weight and AHP
}

\author{
Zhi-Peng Jia, Zhi-Qing Huang \\ Faculty of Information Technology, Beijing University of \\ Technology \\ Beijing Engineering Research Center for IoT Software \\ and Systems, Beijing, China \\ E-mail: s201425029@emails.bjut.edu.cn, \\ zqhuang@bjut.edu.cn
}

\author{
Yan-Xin Zhang \\ College of Electronic Information Engineering, Beijing \\ Jiao tong University, Beijing, China \\ E-mail: yxzhang@bjtu.edu.cn
}

\begin{abstract}
Wireless sensor networks (WSNs) have been widely used in many fields. However, it is difficult to detect WSNs performance status directly. Therefore, a reasonable network performance evaluation system can help to detect and maintain the WSN, so as to ensure the normal operation of the wireless sensor networks. However, the current methods for performance evaluation of wireless sensor networks mainly focus on a single subjective evaluation or objective evaluation, with a certain degree of one-sidedness. Therefore, this paper presents an objective and subjective evaluation model based on the entropy weight method and the AHP to evaluate the throughput, packet loss rate and transmission delay of each path of the WSNs synthetically. According to the experimental results, this model evaluates the network quality of each path in the simulation network systematically, which not only reflects the difference of network quality among different paths, but also provides a solid theoretical foundation for network performance optimization and promotion.
\end{abstract}

Keywords-WSNs; performance evaluation; entropy weight; AHP; comprehensive evaluation

\section{INTRODUCTION}

In recent years, with the rapid development of wireless sensor networks [1], wireless sensor network technology has become the research focus [2] in embedded field, but also the key components of the Internet of things technology and industry 4.0. However, the application of wireless sensor network is also facing many challenges, such as wireless sensor nodes are usually deployed in the harsh environment of the region, in a long time working state, the node energy consumption will continue, which causes the network working condition is difficult to control. Therefore, in order to effectively monitor and improve the quality of the network, it is necessary to evaluate the overall performance of the network state of the whole network to help the user to control the working status of the network in time.

At present, the research on performance evaluation of wireless sensor networks is divided into objective evaluation and subjective evaluation. In objective evaluation, in the literature [3], the authors propose a mathematical analysis method for the analysis and evaluation of the average queue length and waiting time of the data packets in wireless sensor networks with multiple channels and admission control schemes. In literature [4], the author established a real environment, so the wireless sensor network based on 802.15.4 IEEE conducted a preliminary assessment of the performance evaluation indicators, including the data throughput and the received signal strength. In the subjective evaluation such as [5], the authors measured the number of nodes residual energy, the survival of node and the delay in different time, the assignment by the subjective evaluation weights of three indexes, which makes the performance evaluation of wireless sensor network. In [6], the author puts forward the different performance index set an acceptable threshold value, classification of network performance evaluation on the human, and the division of the four scales, and the packet loss rate, repetition rate, load balancing and delay of the four indicators of performance rating, and make the evaluation. In literature [7], author use AHP to solve the wireless sensor network performance index weight distribution by the author, evaluate the storage overhead, communication overhead, connectivity and other aspects, but because the method relies heavily on the experience, has large subjective factors, will have one sidedness.

The various methods mentioned above, the use of the assessment indicators are significant differences, it is difficult to unify. Aiming at this problem, put forward a kind of subjective and objective weighting method based on the decision, not only depends on the subjective decision factors, considering the characteristics of the collected data, using the principle of information entropy in information theory, explore the characteristics of the data itself, the comprehensive performance evaluation on the performance of WSNs. Through the performance evaluation of WSN, it can provide decision-making reference for node redeployment, route selection and potential node failure judgment, so it has obvious application value and research significance.

\section{EVALUATION METHOD OF WSNS BASED ON ENTROPY WEIGHT AND AHP}

In order to build a reasonable network performance evaluation system, we must collect the performance indicator information. At present, the indicator selection has established many mature principles, mainly including following three principles: the comprehensive, easy to test, the relevance. In this paper, three main indicators, delay, packet loss rate and throughput, are selected to evaluate 
WSNs performance. WSNs performance evaluation process is shown in Figure 1.

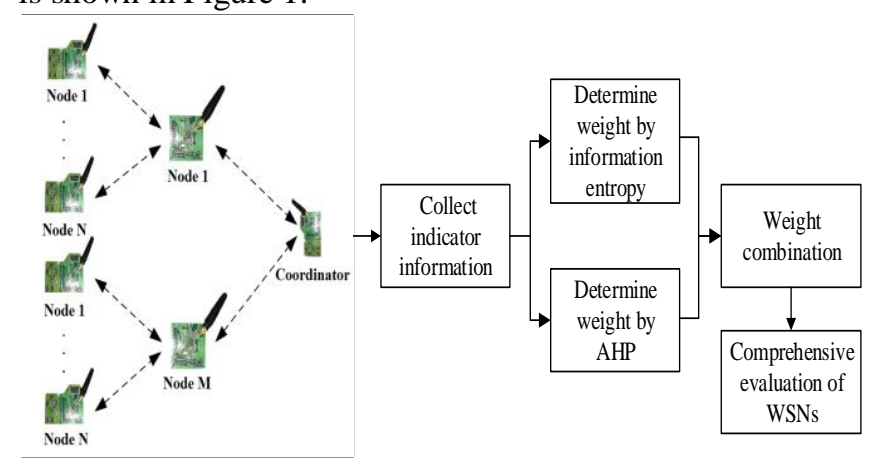

Figure 1. Overall process of WSN performance evaluation.

As can be seen from Figure 1, first, through the coordinator to collect performance indicators on each path. Then respectively using entropy weight method and AHP to calculate the weight of these indicators, then calculate the weight of the two methods combination. Next, combined with the previously mentioned three indicators of WSNs for comprehensive evaluation.

\section{A. Weight Assignment Based on Entropy Weight}

The entropy weight is a kind of objective weighting method, which is used to calculate the corresponding information entropy [8], and then calculate the weight of each indicator by using the information entropy. The entropy weight method is a method that determines the weight by the attribute of the information itself, which belongs to the objective weighting method:

From the wireless sensor network to obtain the sample value and the object to construct a $\mathrm{N} \times \mathrm{M}$ decision matrix $\mathrm{X}$, as shown in the formula (1). The $N=\left(N_{1}, N_{2}, N_{3}, \cdots N_{n}\right)$ represent $\mathrm{N}$ measurement indicators, including delay, packet loss rate and throughput, $M=\left(M_{1}, M_{2}, M_{3}, \cdots M_{m}\right)$ represent for the $\mathrm{M}$ objects to be evaluated. In this paper, the different paths as the objects to be evaluated.

$$
\left[\begin{array}{cccc}
X_{11} & X_{12} & \cdots & X_{1 n} \\
X_{21} & X_{22} & \cdots & X_{2 n} \\
\vdots & \vdots & \ddots & \vdots \\
X_{m 1} & X_{m 2} & \cdots & X_{m n}
\end{array}\right]
$$

Secondly, due to different dimensions of different indicators, so it cannot be directly used to calculate the original decision matrix, need to standardize the raw data, make $0 \leq Y_{m n} \leq 1$. For positive indicators, such as throughput, the formula (2) can be used to carry out the standardization process. Positive indicators (also known as efficiency indicators or look large indicators) are upward or forward indicators of growth, the greater the value of these indicators, the evaluation of the higher quality.

$$
Y_{m n}=\frac{x_{m n}-\min x_{n}}{\max x_{n}-\min x_{n}}
$$

On the other hand, the negative indicators, such as packet loss rate and delay, using the formula (3) for standardization. The so-called negative indicators (also known as the cost index or at small index) is the indicator of value is small, the higher the quality evaluation.

$$
Y_{m n}=\frac{\max x_{n}-x_{m n}}{\max x_{n}-\min x_{n}}
$$

Among them, $Y_{m n}$ represent the normalized value of the $\mathrm{n}$ indicators of $\mathrm{m}$ paths, $\max _{\mathrm{n}}$ represent the maximum of indicator $\mathrm{n}, \min \mathrm{x}_{\mathrm{n}}$ represents the minimum of indicator $\mathrm{n}$.

According to formula (4), calculating the proportion of the $\mathrm{m}$ paths under the indicators of delay, packet loss rate and throughput.

$$
\mathrm{k}_{\mathrm{ij}}=\mathrm{Y}_{\mathrm{ij}} / \sum_{\mathrm{i}=1}^{\mathrm{m}} \mathrm{Y}_{\mathrm{ij}}
$$

Using equation (5) calculate the entropy of indictor delay, packet loss rate and throughput.

$$
\mathrm{E}_{\mathrm{n}}=-\mathrm{L} \sum_{\mathrm{m}=1}^{\mathrm{m}} \mathrm{k}_{\mathrm{mn}} \ln \mathrm{k}_{\mathrm{mn}}
$$

Among them, $\mathrm{L}$ is a constant, $\mathrm{L}=\frac{1}{\ln m}$

The information entropy of indicator directly affects the weight of the all indicators, when the information entropy is large, it means lack of useful information provided by the indicator, when the information entropy is small, it reflects the indicator provides more useful information. Therefore, the definition of $\mathrm{d}_{\mathrm{n}}$ to represent the value of information utility:

$$
\mathrm{d}_{\mathrm{n}}=1-\mathrm{E}_{\mathrm{n}}
$$

To calculate the index weight by entropy method, the essence is the use of the indicators of information utility value to calculate the weight of delay, packet loss rate and throughput. Utility value is greater, the proportion is bigger, the contribution of the evaluation of the results is greater. The formula for calculating the weight of each indicator is:

$$
\mathrm{w}_{\mathrm{n}}=\frac{\mathrm{d}_{\mathrm{n}}}{\sum_{\mathrm{n}=1}^{\mathrm{n}}\left(1-\mathrm{E}_{\mathrm{n}}\right)}
$$

\section{B. Weight Assignment Based on AHP}

AHP is a combination of qualitative and quantitative method for the comprehensive decision making [9]. This method can decompose the complex model into a number of factors, and compare the operation in various factors, thus obtains the weights of different indicator value. As AHP has these advantages, we choose AHP as the subjective decision-making method. The AHP weight assignment process is:

In this paper, the evaluation of network performance is defined as the target layer, the delay, packet loss rate and throughput are defined to the criterion layer, and the network object to be evaluated is put into the solution layer. 
To establish a hierarchical analysis model, the evaluation of network performance is defined as the target layer, the delay, packet loss rate and throughput are defined as the criteria layer, and the paths of WSNs to be evaluated to be defined as the program layer.

To construct the judgment matrix. At the same time, in order to avoid the conflict of multi indicator comparative in judgment matrix, the American scientist Saaty proposed a consistent matrix method, make any of the two indicators of the delay, packet loss rate and throughput compared with each other, not compare all indicator at same time, that can reduce the difficult of multi index compare to improve the accuracy of comparison.

The next step is single hierarchical arrangement, it refers to all of the indicators such as delay, packet loss rate and throughput compared to their previous level(WSNs performance) to get the comparison result, this is require to calculate the maximum eigenvalue and the characteristic vector of three indicators.

It is usually use sum-product method in calculation, first is column vector normalization:

$$
\omega_{\mathrm{ij}}=\mathrm{a}_{\mathrm{ij}} / \sum_{\mathrm{i}=1}^{\mathrm{m}} \mathrm{a}_{\mathrm{ij}}
$$

And then add normalized judgment matrix together by row:

$$
\omega_{i}=\sum_{j=1}^{n} \omega_{i j}
$$

Next, normalization of $\omega$ :

$$
\omega=\omega_{\mathrm{i}} / \sum_{\mathrm{i}=1}^{\mathrm{m}} \omega_{\mathrm{i}}
$$

The $\omega$ represent characteristic vector of the delay, packet loss rate and throughput.

Finally, using formula (11) to get the maximum eigenvalue.

$$
\lambda=\frac{1}{n} \sum_{i=1}^{m} \frac{(A \omega)_{i}}{\omega}
$$

To make consistency check. When the judgment matrix order is too large, it is difficult to make matrix have consistency, if the matrix constructed is not consistent, it will cause errors, so it is necessary to make consistency check use formula (12).

$$
C I=\frac{\lambda-n}{n-1}
$$

When $\mathrm{CI}=0$, with complete consistency, and the $\mathrm{CI}$ closer to the 0 , the higher the consistency; and when the CI is greater, the more serious of inconsistency.

Considering the consistency of the deviation may be caused by random reasons, then introduce the concept of consistency ratio $\mathrm{CR}$ :

$$
C R=\frac{C I}{R I}
$$

$\mathrm{RI}$ as random consistency index. When $\mathrm{CR}<0.1$, it was considered that the degree of inconsistency in the allowable range. When $\mathrm{CR} \geq 0.1$, means it does not meet the consistency check, need to adjust the judgment matrix. If the judgment matrix is passed the consistency check, the characteristic vector of the delay, packet loss rate and throughput is the weight distribution of the different indicator.

\section{DESIGN OF EVALUATION SYSTEM FOR WSNS}

\section{A. Selection of Performance Indicators for WSNs}

In the performance evaluation of the network, usually select several performance indicators into consideration, and not just select one indicator to evaluate. In the selection of the comprehensive performance evaluation indicator, should follow the following three principles [10].

- Overall, the selected indicators do not need much more, but as far as possible to ensure that the overall indicators.

- Easy to measure, the selection of indicators should be easy to measure.

- Correlation, the correlation of the selected indicators should be as small as possible.

After a comprehensive consideration, this paper selects delay, packet loss rate and throughput as the indicators

The delay mainly reflects the time difference of the data packet from the sending to the receiving. When the delay is too large, it will affect the quality of the communication.

Packet loss rate is the ratio of the difference of packets sent and packet received with all packets sent. Packet loss rate too large will lead to the loss of some key data, it will impact the network quality.

Throughput is the maximum rate that the device can accept without the loss of the data frame in the unit time.

\section{B. The Process of Performance Evaluation System for WSNS}

This paper proposes a wireless sensor network performance evaluation system based on entropy weight method and AHP. The main process is shown in Figure 2.

In this evaluation system, first, through the original data collection, combined with the relevant formula mentioned above, based on the information entropy, the decision matrix is constructed, calculate the positive and negative indicators, then get the weights based on the entropy weight method.

Secondly, according to the application requirements to establish the hierarchical demand model and judgement matrix, and combined with the above contents, make single hierarchical arrangement and consistency check, obtain the weights of AHP. 


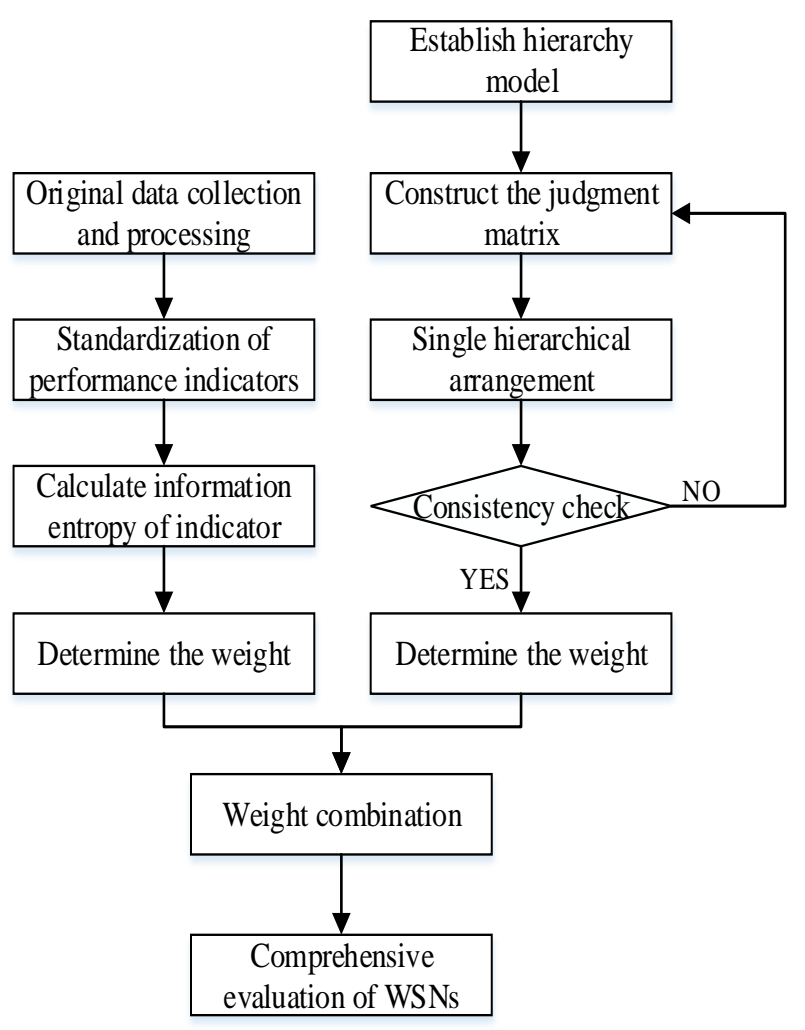

Figure 2. Schematic of evaluation system of WSNs.

Finally, integrated the weight of entropy weight method and AHP through the formula (14), to get the performance evaluation indicator weights of this article.

$$
\omega=\frac{\omega_{1 i} \omega_{2 i}}{\sum_{i=1}^{n} \omega_{1 i} \omega_{2 i}}
$$

\section{NETWORK PERFORMANCE EVALUATION AND RESULT ANALYSIS}

\section{A. Experimental Environment}

In this paper, OPNET simulation software is used to build the simulation platform. OPNET simulation software can accurately analyze the performance and behavior of complex networks, and provides a series of simulation models, which can be easily for data collection and statistical analysis. In this paper, the Zigbee model is used to implement at the OPNET in this experiment. The network structure of the deployment of 60 nodes to simulate the Zigbee network, at the same time using the Mesh network topology which is widely applied, and ensure that all nodes in the communication range, the simulation of 30 minutes. The simulation schematic is shown in Figure 3.

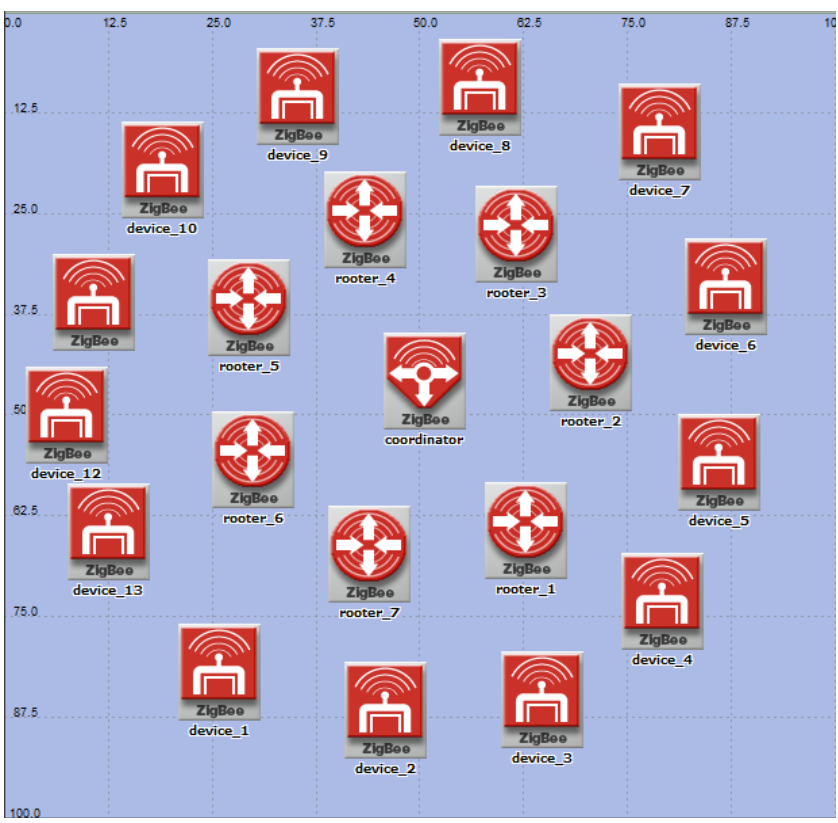

Figure 3. Schematic of WSNs simulation.

\section{B. Network Performance Evaluation and Analysis}

First of all, this paper selects the 6 paths of the measurement analysis, and select tenth minutes, twentieth minutes of the measured values and 30 minutes of the average data for statistical analysis, statistical results is shown in the table I.

Then, the obtained data are analyzed according to the entropy weight decision model proposed in the previous paper. In this experiment, delay and packet loss rate are all negative indicator, the smaller the better; and the throughput belongs to the positive indicator, the bigger the better; so in accordance with this standard to standardize original data, then through the information entropy formula get the entropy of three indicators were $[0.91,0.95,0.87]$, and to calculate the weights of three indicators were $[0.34,0.20,0.46]$.

From the above results, we can find that the weight of the throughput in the three indicators is the largest, with a greater distinction, and the proportion of the packet loss rate is relatively small, the distinction is relatively small.

Secondly, using the AHP, the three indicators are compared, and the delay is regarded as the most important index, and the packet loss rate is the second, but it is better than the throughput, so the judgment matrix can be obtained:

TABLE II. JUDGEMENT MATRIX

\begin{tabular}{|c|c|c|c|}
\hline & Delay & Packet loss rate & Throughput \\
\hline Delay & 1 & 2 & 3 \\
\hline Packet loss rate & $1 / 2$ & 1 & 2 \\
\hline Throughput & $1 / 3$ & $1 / 2$ & 1 \\
\hline
\end{tabular}

Put the judgment matrix into AHP model, through the calculation of the characteristic vector and maximum eigenvalue, the characteristic vector is $[0.54,0.30,0.16]^{\mathrm{T}}$, maximum eigenvalue is $\lambda=3.009$. 
According to the above results, the consistency check is obtained

$$
C R=\frac{C I}{R I}=0.008
$$

According to the result of the consistency check, the judgment matrix through the consistency check, the weight of three indicators determined by AHP is $[0.54,0.30,0.16]$.

Finally, according to the formula (14), we can get the comprehensive weight value of three indicator at this experiment is [0.58, 0.19, 0.23].

Now, we can evaluate the network performance of different paths according to the obtained weight value, and the evaluation results are shown below:

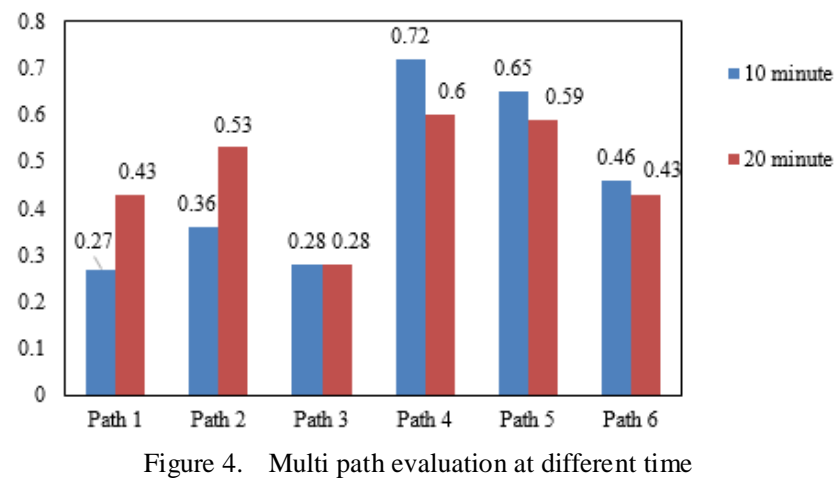

As can be seen from Figure 4, path 4 at 10 minutes and 20 minutes the score is relatively high. Through the analysis of the original data can be learned, this is mainly due to the delay of path 4 in the two time is smaller, and the delay is the largest comprehensive weight of this performance evaluation which leads to the score of path 4 is relatively high, but the evaluation of the two moment is different, this is because at 10 minutes, the packet loss rate of the path 4 is far lower than the packet loss rate at 20 minutes, and the throughput at two times is basically unchanged, which leads to the performance of the path 4 in 10 minutes when the performance is better than the time of 20 minutes.

The path 2 due to the delay in the 20 minute delay is less than 10 minutes, at the same time with the least of all the data packet loss rate, although the throughput in 10 minutes is slightly higher than the 20 minutes throughput, but due to the weight of the packet loss rate and throughput is close, the throughput of path 2 advantages in 10 minutes are not significant to improve the performance of path 2 in the 10 minute, which makes the performance of the path 2 in the 20 minute is better than the performance of 10 minute.

Figure 5 shows the average value of the performance evaluation of the 6 paths within 30 minutes, from the graph can be learned that path $4,5,6$ of the evaluation values in 10 minutes and 20 minutes two times better than path 1,2,3.

Through the analysis of the original data can be learned, this is mainly because the path of the $4,5,6$ of the delay is far less than the path $1,2,3$, and through the comprehensive evaluation of entropy weight method and AHP, the weight value of the delay indicator in the three indicator has the largest proportion, which leads to the performance of the path $4,5,6$ on the whole is better than the path $1,2,3$.

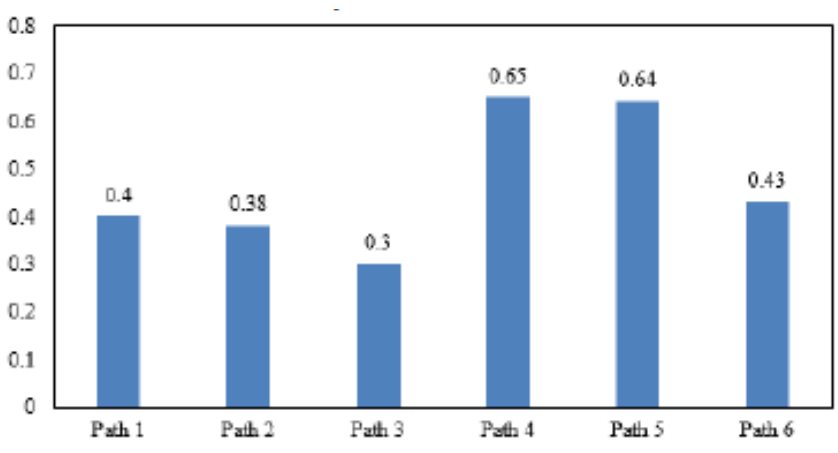

Figure 5. Multi path 30 min average performance.

The lower overall performance of path 2,3 compared to others is due to their delay is too high, which leads to the performance evaluation value of the 2 paths is relatively low, but the throughput of path 2 is larger than path 3 , which makes the path performance evaluation value of 2 slightly higher than the path 3 .

\section{CONCLUSION}

In this paper, a general performance evaluation model based on the entropy weight method and the AHP is proposed to solve the problem of the lack of a general performance evaluation model in the wide application of wireless sensor networks. The model has the advantages of high accuracy, strong adaptability and clear structure, which makes up the deficiency of single evaluation method. Through experiments, this model can accurately analyze the quality differences of different network paths, and lays a solid foundation for improving network performance.

At the same time, the model can be applied to a variety of wireless sensor network applications by modifying the parameters of the judgment matrix, which can be used to evaluate the performance of different wireless sensor networks.

\section{ACKNOWLEDGMENT}

This work is supported in part by National Development and Reform Commission (NDRC) (No. Q5025001201502), the Fundamental Research Funds for the Central Universities (No. W16JB00340) and the BJUT graduate excellent course construction project (No. 025000514116506).

\section{REFERENCES}

[1] Ren FY, Huang HN, Lin C. Wireless sensor networks. Journal of Software, 2003,14(7):1282 1291.

[2] Huang P, Xiao L, Soltani S, et al. The Evolution of MAC Protocols in Wireless Sensor Networks: A Survey[J]. IEEE Communications Surveys \& Tutorials, 2013, 15(1):101-120.

[3] Ma Z, Yue W, Tian N. Performance evaluation of wireless communication networks for multi-traffic with multi-channel[C]//2009 IEEE Wireless Communications and Networking Conference. IEEE, 2009: 1-6.

[4] Lee J S. An experiment on performance study of IEEE 802.15. 4 wireless networks[C]//2005 IEEE Conference on Emerging Technologies and Factory Automation. IEEE, 2005, 2: 8 pp.-458.

[5] Lingyun Y, Xingchao W. Study on performance evaluation method based on measurement for wireless sensor network [C] // 
Communications Technology and Applications, 2009. ICCTA'09. IEEE International Conference on. IEEE, 2009: 201-206.

[6] Yan X, Wang F, Hu L, et al. Application-oriented Network Performance Evaluation for the Internet of Things[J]. International Journal of Online Engineering, 2013.

[7] Ruan N, Ren Y, Hori Y, et al. Performance analysis of key management schemes in wireless sensor network using analytic hierarchy process[C]//2011IEEE 10th International Conference on
Trust, Security and Privacy in Computing and Communications. IEEE, 2011: 1739-1744.

[8] Shannon C E. A mathematical theory of communication[M]// Mathematical theory of computation /. McGraw-Hill, 1974:3-55.

[9] Saaty T L. How to make a decision: The analytic hierarchy process[J]. European Journal of Operational Research, 1990, 48(1):9-26.

[10] Zhang D Y, Hu M Z, Zhang H L. Study on network performance evaluation method based on measurement[J]. JOURNAL-CHINA INSTITUTE OF COMMUNICATIONS, 2006, 27(10): 74

TABLE I. NETWORK PERFORMANCE MEASUREMENT RESULTS

\begin{tabular}{|c|c|c|c|c|c|c|c|c|c|}
\hline & \multicolumn{2}{|c}{ Delay (ms) } & \multicolumn{2}{|c|}{ Packet loss rate (\%) } & \multicolumn{2}{c|}{ Throughput (bits/s) } & \multicolumn{3}{c|}{ Average } \\
\hline Path & 10 minute & 20 minute & 10 minute & 20 minute & 10 minute & 20 minute & Delay & Packet loss rate & Throughput \\
\hline Path 1 & 34.007 & 30.964 & 13.88887 & 8.333301 & 5497.555 & 5300.501 & 32.882 & 7.888887 & 5530.542 \\
\hline Path 2 & 36.496 & 33.935 & 11.11113 & 2.777734 & 6691.556 & 6313.334 & 37.027 & 8.66667 & 6647.973 \\
\hline Path 3 & 39.132 & 37.883 & 4.242578 & 7.058398 & 5497.556 & 5582.389 & 36.747 & 7.777773 & 5576.053 \\
\hline Path 4 & 19.434 & 19.298 & 5.555566 & 12.91777 & 4327.278 & 4380.111 & 20.578 & 8.111113 & 4438.911 \\
\hline Path 5 & 23.28 & 24.262 & 10.1291 & 11.1708 & 5561.111 & 5464.056 & 23.805 & 9.388887 & 5485.4 \\
\hline Path 6 & 27.931 & 26.584 & 6.814258 & 9.933789 & 4435.389 & 4309.278 & 27.835 & 8.388887 & 4396.342 \\
\hline
\end{tabular}

